\title{
Profiles and Clinical Outcomes of Perforated Peptic Ulcer with Boey Score 3 Treated with External Drainage in Indonesia
}

\author{
Rachman Efendi ${ }^{1}$, Denny Septarendra ${ }^{2}$ \\ ${ }^{1}$ Department of Surgery, Faculty of Medicine Universitas Airlangga \\ Dr. Soetomo General Hospital, Surabaya, Indonesia \\ ${ }^{2}$ Digestive Surgery Division, Department of Surgery, Faculty of Medicine Universitas Airlangga \\ Dr. Soetomo General Hospital, Surabaya, Indonesia
}

*Corresponding author details: Rachman Efendi, M.D.; ma2n.eph@gmail.com

\begin{abstract}
The treatment of peptic ulcers has changed substantially, and the rate of elective surgery for gastroduodenal peptic ulcers has fallen significantly; as a result, the rate of emergency surgery for perforated peptic ulcers has slightly increased. The Boey score is one of the most widely used prognostic scoring systems for predicting death in peptic ulcer perforation patients. The Boey scoring system has a positive connection with mortality risk. Patients with Boey Score 3 are patients who meet the high-risk criteria and have high morbidity and mortality rates. Conservative therapy can be utilized as an alternative to surgery in some cases with perforated gastroduodenal ulcers. In this study, we presented a clinical case of perforated peptic ulcer patient with Boey score 3 treated with external drainage for the conservative treatment.
\end{abstract}

Keywords: perforated peptic ulcer; Boey score; external drainage; clinical outcomes

\section{INTRODUCTION}

Peptic ulcer perforation is a disease caused by a discontinuity of the gastric or duodenal mucosal wall that spreads to the submucosa or deeper, which results in leakage into the abdominal cavity. Duodenal ulcer perforation is reported to be 2-3 times more common than gastric ulcer perforation. Peptic ulcer perforation is characterized by acute abdominal pain and followed by high risk of morbidity and mortality 1 . Every year, peptic ulcer disease affects 4 million people worldwide, $10-20 \%$ of patients with peptic ulcer develop complications and about $2-14 \%$ will have a tendency to perforate. The incidence of peptic ulcer perforation is quite rare, but has a high mortality (5-25\%) and rising to as high as 50\% with age 2 .

In Indonesia, the prevalence of peptic ulcer ranges from 6$15 \%$. Peptic ulcer perforation remains as one of the common causes of death in men aged 45-54 years (Koto, Asrul and A, 2016). Patients with peptic ulcer perforation who have undergone surgery still have high risk factors for morbidity (20\%-54.5\%) and mortality (3\%-40\%). Currently, peptic ulcer perforation is still a challenge for surgeons and requires optimal treatment strategies to provide good outcomes for patients 3 .

The Boey score is one of the prognostic scoring systems commonly used to predict the mortality in patients with peptic ulcer perforation. Previous study reported that the Boey scoring system had a positive correlation with the risk of mortality (Boey score $0=0 \%$; Boey score $1=37.5 \%$, Boey score $2=52.9 \%$, and Boey score $3=100 \%$ ), however, the Boey scoring system was not significantly associated with the risk of patient death $(\mathrm{P}>0.05)$ 4. Currently, external drainage is widely used by surgeons in clinical practice to treat patients with perforated peptic ulcer, especially with Boey score ${ }^{3}$. Previous study reported that external drainage was effective as a management for perforated peptic ulcer with Boey score 3, and this method reduced the mortality rate 5 .
It is expected that $40-80 \%$ of peptic ulcer perforations will close spontaneously with conservative therapy (Taylor's Method) so that peptic ulcer perforations that close spontaneously do not need laparotomy. Non-operative therapy with external drainage combined with conservative management such as Taylor's method had higher success rate compared to laparotomy ( $47 \%$ vs. $20 \%$, respectively) 6 .

Currently, there was limited study examining the general profiles and the outcome of patients with peptic ulcer Boey score 3 treated with external drainage. Therefore, in this study we aimed to analyze the profile, distribution, and clinical outcome of patients with Boey score 3 in Indonesia.

\section{MATERIALS AND METHODS \\ Study design}

The design of this study was a retrospective cohort observational descriptive study to provide an overview of the mortality rate of patients with peptic ulcer perforation Boey Score 3 in the Digestive Surgery division, Department of Surgery, Faculty of Medicine Universitas Airlangga - Dr. Soetomo General Hospital (Surabaya, Indonesia). We collected the data of patients with peptic ulcer perforation Boey Score 3 from the medical records data from the visit to the Emergency Department, Dr. Soetomo General Hospital (Surabaya, Indonesia) from January 2019 to December 2021. In this study, we included peptic ulcer perforated patient with Boey Score 3 who underwent external drainage. We excluded patients with perforated peptic ulcer who died before external drainage was performed. The patient's data, such as name, age, gender, address, and outcome were recorded for further analyses.

\section{Statistical analysis}

The statistical analysis was performed using the SPSS statistical software package version 23.0 (IBM Corp., Armonk, NY, USA). 


\section{RESULTS}

\section{Subject characteristics}

We included a total of 56 subjects, consisted of 37 men $(37 / 56 ; 66.1 \%)$ and 19 women $(19 / 56 ; 33.9 \%)$. The youngest subject was 26 years old and the oldest was 79 years old. The mean age was $61.4 \pm 12.8$ years old. The age distribution of the study subjects is shown in Table 1 . Based on the age group, we found that the highest prevalence of perforated peptic ulcer was occurred between $61-70$ years old $(17 / 57 ; 36.8 \%)$, followed by 71 80 age group (30.4\%), 51-60 age group (14/56; $25.0 \%)$. The occurrence was the lowest in subject in 31-40 years group. Based on the outcomes, there were 41 subjects $(41 / 56 ; 73.2 \%)$ who survived after treatment and 15 subjects $(15 / 56 ; 26.8 \%)$ who were not survive after receiving treatment.

TABLE 1: The age distribution of the study subjects

\begin{tabular}{lcr}
\hline \multicolumn{1}{c}{ Characteristic } & n & Percentage \\
\hline Age & 3 & \\
$21-30$ & 1 & $5.4 \%$ \\
$31-40$ & 6 & $1.8 \%$ \\
$41-50$ & 14 & $25.0 \%$ \\
$51-60$ & 17 & $36.8 \%$ \\
$61-70$ & 15 & $30.4 \%$ \\
$71-80$ & & \\
Sex & 37 & $66.1 \%$ \\
Male & 19 & $33.9 \%$ \\
Female & & \\
Outcomes & & $73.2 \%$ \\
Survive & 41 & $26.8 \%$ \\
Dead & 15 &
\end{tabular}

\section{DISCUSSION}

The Boey score scoring system is one of the prognostic prediction systems for patients with perforated peptic ulcer. This system helps in predicting patient's outcome because in general, patients with perforated peptic ulcer have high morbidity and mortality rates 7 . In this study, we analyzed patients with peptic ulcer perforation with Boey score 3. Based on age, we found that the prevalence of peptic ulcer perforation was more common in male than in female. Our result was in accordance with previous study which also reported that male subjects were at higher risk for peptic ulcer perforation ${ }^{8}$. This phenomenon might be related to the fact that hormone estrogen protects the gastric mucosa by increasing bicarbonate levels 9,10 .

Based on the age distribution, the highest number was found between 61-70 years and followed by 71-80 age group. These data indicate that the incidence of peptic ulcer perforation is associated with increasing age, e.g. over the age of 60 years. The increasing age is associated with the increase in the incidence of degenerative diseases which requiring treatment with NSAIDs, where this treatment is known to be the cause of the incidence of peptic ulcer perforation 11 .

Based on the distribution of patients with peptic ulcer perforation with a Boey score of 3 (three) who underwent external drainage, it was found that the most patients experienced mortality as many as 41 patients $(73.2 \%)$ followed by living patients as many as 15 patients $(26.8 \%)$. From the results of this study, it can be concluded that the mortality rate is $73.2 \%$. This is because patients with a Boey score of 3 are patients who have a very high surgical risk such as the onset of perforation more than 24 hours, preoperative blood pressure and comorbidities that the patient has. Where the onset of perforation is more than 24 hours and preoperative blood pressure plays a major role in influencing mortality. Meanwhile, the patient's comorbidities slightly affect the occurrence of mortality.
The onset of perforation was determined based on the onset of acute abdominal pain. As the duration of perforation increases, severe bacterial contamination occurs. The higher the bacterial contamination and proliferation of the peritoneum, the higher the severity of peritonitis, the worse the prognosis. In addition, the longer duration of peritonitis also affects the occurrence of sepsis in patients. This septic condition plays a role in the mortality of patients with perforated peptic ulcer. Preoperative blood pressure in question is a state of shock experienced by preoperative patients. Preoperative shock is caused by dehydration due to decreased fluid intake and sepsis arising from peritonitis. These two things will burden each other so that it decreases hemodynamics and increases injury to other people. The patient's comorbidity is not directly related to the mortality of patients with peptic ulcer perforation. Several diseases such as heart disease, hypertension, lung disease have been reported to have a high morbidity rate in patients with peptic ulcer perforation 12 .

Patients with a Boey score 3 was defined as patients with delays in medical action $>24$ hours, the presence of shock, and the presence of comorbidities that could aggravate the patient's condition. Thus, patients with Boey Score 3 are patients who meet the high-risk criteria and have high morbidity and mortality rates. The condition of high-risk patients can make the decision to operate more difficult due to the possibility of complications during surgery. Therefore, external drainage can be considered as a therapeutic option in patients with Boey score of 3 . Previous studies suggested the use of external drainage methods in patients with perforated peptic ulcer who are at high risk 13 .

Several studies have been conducted to examine the clinical outcome of external drainage as a treatment in patients with perforated peptic ulcer. Saber et al conducted a study of the use of external drainage in patients with high-risk peptic ulcer perforation, which resulted in satisfactory results with a mortality rate of $20.8 \%$ 13. Meanwhile, Oida et al in Japan found that in the group treated external drainage there were 3 patients $(14.3 \%)$ who died while the group without external drainage found 13 patients (43.3\%) who died ${ }^{5}$

The consideration of the non-operative management (NOM) method with external drainage in Boey score 3 patients is to reduce the burden or stress caused by surgery. Surgical procedure puts an excessive burden on the patient's body with a Boey score of 3 by suppressing organ function which had actually been reduced before surgery. External drainage is intended for source control and intraperitoneal lavage, and has a better outcome in patients with peptic ulcer perforation at high risk or Boey score 3. Studies have shown that approximately $40 \%-80 \%$ of gastric perforations caused by peptic ulcer will close spontaneously with conservative management. External drainage should be combined with adequate general supportive care such as fluid therapy, antibiotics, and antigastric acid secretion drugs (PPIs or $\mathrm{H} 2$ blockers). There are several components Taylor's method in peptic ulcer perforation, such as: (1) gastric emptying (NGT insertion and active suction); (2) antibiotics; (3) H2 blockers, PPIs and mucoprotectants; (4) vital sign monitoring and fluid balance; and (5) access to operation room ${ }^{14}$. The mortality rate for non-operative management in patients with closed perforations was $3 \%$ compared with $6.2 \%$ where emergency surgery was performed. This suggests that gastric perforation with closed perforation can be managed conservatively. 
In clinical practice, non-operative management strategies are resource-intensive and require a commitment to active clinical examinations along with the availability of a surgeon at all times and if there is a clinical deterioration, and emergency surgery is required. Thus, external drainage can be recommended as a treatment in patients with perforated peptic ulcer with a Boey score of 3 or at high risk.

\section{CONCLUSION}

The success rate of external drainage in patients with perforated peptic ulcer with a Boey score 3 in Dr. Soetomo General Hospital was 26.8\%. Comprehensive nonoperating management is important to increase the survival rate of patients with peptic ulcer perforation Boey score 3 or at high risk.

\section{CONFLICTS OF INTEREST}

No competing interests declared.

\section{REFERENCE}

[1] Taş İ, Ülger BV, Önder A, Kapan M, Bozdağ Z. Risk factors influencing morbidity and mortality in perforated peptic ulcer disease. Ulus cerrahi Derg. 2015;31(1):20-5.

[2] Christensen S, Riis A, Nørgaard M, Sørensen HT, Thomsen RW. Short-term mortality after perforated or bleeding peptic ulcer among elderly patients: a population-based cohort study. BMC Geriatr. 2007 Apr; 7:8.

[3] Søreide K, Thorsen K, Søreide JA. Strategies to improve the outcome of emergency surgery for perforated peptic ulcer. 2014;51-64.

[4] Nuzulistina E, Putro MD, Fauziah D. Evaluating Outcome in Perforated Peptic Ulcer by Boey and POMPP Score. JUXTA J Ilm Mhs Kedokt Univ Airlangga. 2021; XII (01).

[5] Oida T, Kano H, Mimatsu K, Kawasaki A, Kuboi Y, Fukino N, et al. Percutaneous drainage in conservative therapy for perforated gastroduodenal ulcers. Hepatogastroenterology. 2012;59(113):168-70.
[6] Chung KT, Shelat VG. Perforated peptic ulcer - an update. World J Gastrointest Surg. 2017 Jan;9(1):1-12.

[7] Agarwal A, Jain S, Meena LN, Jain SA, Agarwal L. Validation of Boey's score in predicting morbidity and mortality in peptic perforation peritonitis in Northwestern India.

Trop Gastroenterol. 2015;36(4):256-60.

[8] Bertleff MJOE, Lange JF. Perforated peptic ulcer disease: a review of history and treatment. Dig Surg. 2010 Aug;27(3):161-9.

[9] Tuo B, Wen G, Wei J, Liu X, Wang X, Zhang Y, et al. Estrogen regulation of duodenal bicarbonate secretion and sex-specific protection of human duodenum. Gastroenterology. 2011 Sep;141(3):854-63.

[10] Smith A, Contreras C, Ko KH, Chow J, Dong X, Tuo B, et al. Gender-specific protection of estrogen against gastric acid-induced duodenal injury: stimulation of duodenal mucosal bicarbonate secretion. Endocrinology. 2008 Sep;149(9):4554-66.

[11] Drini M. Peptic ulcer disease and non-steroidal antiinflammatory drugs. Aust Prescr. 2017 Jun;40(3):91-3.

[12] Sivaram P, Sreekumar A. Preoperative factors influencing mortality and morbidity in peptic ulcer perforation. Eur J trauma Emerg Surg Off Publ Eur Trauma Soc. 2018 Apr;44(2):251-7.

[13] Saber A, Gad MA, Ellabban GM. Perforated duodenal ulcer in high risk patients: Is percutaneous drainage justified? N Am J Med Sci. 2012;4(1):35-9.

[14] Anbalakan K, Chua D, Pandya GJ, Shelat VG. Five-year experience in management of perforated peptic ulcer and validation of common mortality risk prediction models - Are existing models sufficient? A retrospective cohort studies. Int J Surg. 2015; 14:38-44. 